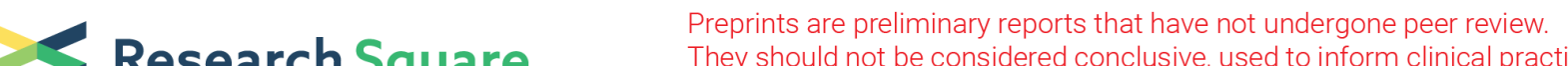 $\begin{array}{ll}\text { Research Square } & \text { They should not be considered conclusive, used to inform clinical practice, } \\ \text { or referenced by the media as validated information. }\end{array}$
}

\section{Walnut Meal Extracts Rich in Polyphenols Mitigate Insulin Resistance and Modulate the Gut Microbiota of High Fat Diet-fed Rats}

\section{Xingping He}

Yunnan University of Traditional Chinese Medicine

\section{Dan Chen}

Yunnan Institute of Tobacco Quality Inspection and Supervision

\section{Dingfa Yang}

Yunnan University of Traditional Chinese Medicine

\section{Xi Zhang}

Yunnan University of Traditional Chinese Medicine

\section{Yage Ma}

Yunnan University of Traditional Chinese Medicine

\section{Jingjuan Yang}

Yunnan University of Traditional Chinese Medicine

\section{Chaoyin Chen}

Yunnan Academy of Forestry

Shenglan Zhao ( $\sim$ zsl13330431529@126.com )

Yunnan University of Traditional Chinese Medicine https://orcid.org/0000-0001-8817-5996

\section{Research}

Keywords: walnut meal polyphenols; adjusting lipidemia; prandial glucose regulator; improve intestinal flora; obese rat trial

Posted Date: April 5th, 2021

DOI: https://doi.org/10.21203/rs.3.rs-355579/v1

License: (c) (i) This work is licensed under a Creative Commons Attribution 4.0 International License. Read Full License 


\section{Abstract}

Background: This paper aims to investigate the metabolic impact of walnut meal extracts rich in polyphenols (WMP) on high fat diet (HFD)-fed rats and to determine whether the lipid-lowering effects are related to modulations of the gut microbiota. SD rats were fed a standard chow diet or an HFD for 18 weeks. After 6 weeks, the HFD rats were supplemented with $750 \mathrm{mg} \mathrm{WMP} / \mathrm{kg}$ body weight and the vehicle for 12 weeks. The structure of gut microbiota was assessed by analyzing 16S rDNA sequences.

Results: WMP suppressed the weight gain and visceral obesity. WMP treatment also improved lipid profiles and increased antioxidative activities. WMP fully reversed hepatic steatosis with upregulation of adipocytokines involved in lipid catabolism (e.g. adiponectin, PPAR-y, visfatin, CEBPa), and increased the activity of lipoprotein lipase, hormone-sensitive lipase, in which were associated with glucose tolerance improved and insulin resistance mitigated. As revealed by 16S rDNA sequencing, WMP restored the diversity of intestinal flora reduced by HFD, dramatically reduced the abundance of Fusobacterium varium and Enterobacteriaceae, reversed and sharply raised the abundance of Lachnospiraceae UCG005 and Akkermansia decreased by HFD.

Conclusion: Our findings demonstrated that WMP suppressed the weight gain and adiposity in HFD-fed rats, and fully reversed HFD diet-induced insulin resistance and hepatic steatosis while dramatically reduced the abundance of Fusobacteriaceae and Enterobacteriaceae, underscoring the gut-liver axis as a primary target of walnut polyphenols.

\section{Introduction}

Obesity is a medical symptom in which excess body fat accumulates to the extent that it may have an adverse effect on health [1]. It increases the risk of cardiopathy, type 2 diabetes, dyslipidemia, hypertension, infections or cancer, osteoarthritis, respiratory or gynecological problems [2]. It occurs owing to an imbalance between energy intake and expenditure, resulting in subsequent excess adipose accumulation [3]. Also, more evidences support the intention that obesity is related with a chronic inflammation based on the abnormal cytokines such as IL-6 and TNF-a, along with the activation of proinflammatory signaling pathways [4]. Insulin resistance (IR) is a physiological status which major peripheral organs like the adipose tissue, liver, and skeletal muscle becomes less sensitive to the action of insulin [5]. It is a usual cause of carbohydrate and lipid metabolic disorders, and often precedes the onset of hyperglycemia and forecasts the development of fatty liver and type 2 diabetes [6].

The gut is habitat to trillions of microbes that have fundamental roles in many aspects of human physiology, including metabolism and immune function [7], and recent evidence proposes that it contributes to the development of obesity and metabolic diseases. Studies have shown that obesity can make the structure of bacteria disorder, and intestinal flora can further increase the intake of dietary energy to promote obesity, it is a necessary condition for high fat diet (HFD) to induce obesity [8-10]. Moreover, HFD was known to induce enhances in intestinal permeability and increases in the secretion of 
intestinal pro-inflammatory cytokines [11]. The intestinal microflora lipopolysaccharide (LPS), a potent inducer of inflammation, plays a key role in the occurrence and development of related metabolic diseases. It is one of the components of the cell wall of some Gram's negative bacteria, and the release of Gram's negative bacteria after death is dissolved. Studies have shown that polyphenols can increase the abundance of probiotics in the intestines, for example, Akkermansia associated with the prevention of the obesity can modulate the structure of bacteria disorder in intestinal flora and further increase the intake of dietary energy to promote abrogation of intestinal inflammation in these animals, so as to achieve the effect of anti-obesity $[12,13]$.

As an abundant of significant nutrients that seem to have potential effects on human health [14], walnut kernel was contained in the Chinese Pharmacopoeia [15]. The walnut consumption presented the cardiometabolic protective effect for obese people [16]. Walnut meal (WM), as a by-product left behind oil, is prepared from the walnut kernel by cold press. It is usually prepared walnut pastry, walnut milk and feed, but usage is limited because of its bitter and astringent [17]. WM is rich polyphenolic components (6.75\%), such as rutin, gallic acid, p-hydroxybenzoic acid, 3-coumaric acid, chlorogenic acid and kaempferol-3-glucoside $[18,19]$. Our previous studies showed that walnut meal extracts performed a strong effect on lowering hyperlipidemia [20]. This paper aims at revealing the effect of WMP on HFD induced metabolic disorders in rats and detecting how the WMP benefits are associated with changes in the intestinal flora.

\section{Materials And Methods}

\section{Animals}

140-160g SD rats ( $n=30$, Hunan, China) were bred, eight rats/cage, in the experimental animalhouse of the Yunnan University of Traditional Chinese Medicine. Animals were housed at a constant temperature of $22 \pm 2{ }^{\circ} \mathrm{C}$ with a relative humidity of $55 \pm 5 \%$ and an artificial $12-\mathrm{h}$ light-darkness cycle. After acclimatization to the experimental environment for one week and on a normal control diet (low-fat diet AIN-93; $10 \%$ energy from palm oil) for a week before experiment, the rats were randomly assigned to three groups including one group continued to be on normal control diet (assigned as NCD, keep feeding it to the end of the experiment) and two high-fat diet groups (AIN-93 adapted; $60 \%$ of energy from palm oil) for 5 weeks, at this time, the serum TC and TG of the two HFD-fed rats were significantly higher than the NCD-fed rats. After 5 weeks, HFD-fed rats were randomly assigned to two groups: one (assigned as WMP) received daily dose $(750 \mathrm{mg} / \mathrm{kg}$ ) of WMP by gavage, whereas another one (assigned as HFD), HFD and NCD received the vehicle (saline). During the experiments, food intake was recorded daily and body weight was recorded weekly. Feces were collected weekly and stored at $-80{ }^{\circ} \mathrm{C}$. Blood samples were gotten from the abdominal aorta, clotted at $37^{\circ} \mathrm{C}$ for $2 \mathrm{~h}$ and centrifuged at $3000 \mathrm{~g}$ for $10 \mathrm{~min}$, the serum was isolated and stored at $-80^{\circ} \mathrm{C}$, and liver weight, body length and fat weight were measured.

\section{Preparation of the polyphenol-enrich extracts from cold pressed degreased walnut meal (WMP)}


The polyphenol extracts (WMP) of walnut meal were extracted in $50 \%$ ethanol $(1: 50, \mathrm{v} / \mathrm{v})$ by reflux extraction for $60 \mathrm{~min}$ at $73{ }^{\circ} \mathrm{C}$, after filtering, sample solution was concentrated under reduced pressure and then lyophilized to obtain a brown powder.

\section{Glucose homeostasis}

At week 12, rats were $12 \mathrm{~h}$ fasted and insulin tolerance tests (ITT) were conducted after an intraperitoneal injection of insulin ( $0.75 \mathrm{Ul} / \mathrm{kg}$ body weight). Blood glucose concentrations were measured with automatic glucose analyzer (Anwen, Sannuo Ltd, Changsha, China) before ( $0 \mathrm{~min})$ and after $(30,60$ and $120 \mathrm{~min}$ ) insulin injection. At the end of week 12, rats were fasted overnight and an oral glucose tolerance test (OGTT) was conducted after gavage with glucose ( $3 \mathrm{~g} / \mathrm{kg}$ body weight). Blood sample was gotten before ( $0 \mathrm{~min})$ and after $(15,30,60,90$ and $120 \mathrm{~min}$ ) glucose challenge for glycaemia detection. Additionally, blood samples $(30 \mu \mathrm{L})$ were gotten at each time point during OGTT for insulin aemia and Cpeptide determination. The homeostasis model assessment of insulin resistance (HOMA-IR) index was calculated as reference described [21].

\section{Biochemical analysis}

Triglyceride(TG), Total cholesterol (TC), low density lipoprotein (LDL-C), high density lipoprotein (HDL-C), glutathione (GSH), malondialdehyde (MDA), glucose, annexin detection kits were bought from Nanjing KenGENBioTECH Corp., Ltd(Nanjing, China). Leptin, adiponectin, insulin, PPAR-y, visfatin, CEBPa, lipoprotein lipase (LPL), hormone-sensitive lipase (HSL) were detected using ELISA kit (Jingmei Bio, Jiangsu, China) according to the manufacturer's protocols.

\section{Microbiota analysis by $16 \mathrm{~S}$ rDNA sequencing}

Microbial DNA was extracted from fecal samples using the E.Z.N.A.® Soil DNA Kit (Omega Bio-tek, Norcross, GA, U.S.) according to the manufacturer's protocols. The V4-V5 regions of the bacteria $16 \mathrm{~S}$ ribosomal RNAgene were amplified by PCR $\left[95^{\circ} \mathrm{C}\right.$ for $2 \mathrm{~min}$, followed by 25 cycles $\left(95^{\circ} \mathrm{C}\right.$ for $30 \mathrm{~s}, 55^{\circ} \mathrm{C}$ for $30 \mathrm{~s}, 72{ }^{\circ} \mathrm{C}$ for $30 \mathrm{~s}$ ) and a final extension at $72{ }^{\circ} \mathrm{C}$ for $\left.5 \mathrm{~min}\right]$ using primers $515 \mathrm{~F} 5$ '-barcodeGTGCCAGCMGCCGCGG)-3' and 907R 5'-CCGTCAATTCMTTTRAGTTT-3', where barcode is an eight-base sequence unique to each sample. PCR reactions were performed in triplicate $20 \mu \mathrm{L}$ mixture containing $4 \mu \mathrm{L}$ of $5 \times$ FastPfu Buffer, $2 \mu \mathrm{L}$ of $2.5 \mathrm{mM}$ dNTPs, $0.8 \mu \mathrm{L}$ of each primer $(5 \mu \mathrm{M}), 0.4 \mu \mathrm{L}$ of Fast Pfu polymerase, and $10 \mathrm{ng}$ of template DNA. Amplicons were isolated from $2 \%$ agarose gels and purified using the AxyPrep DNA Gel Extraction Kit (Axygen Biosciences, Union City, CA, U.S.) according to the manufacturer's protocols and quantified using QuantiFluor ${ }^{\mathrm{TM}}$-ST (Promega, U.S.). Purified amplicons were pooled in equimolar and paired-end sequenced $(2 \times 300)$ on an Illumina MiSeq platform according to the manufacturer's instructions. The raw reads were deposited into the NCBI Sequence Read Archive(SRA) database. Raw fastq files were quality-filtered, demultiplexedusing QIIME (version 1.9.1) with the following criteria: (a) The 300 bp reads were truncated at any site receiving an average quality score $<20$ over a $50 \mathrm{bp}$ sliding window, discarding the truncated reads that were shorter than $50 \mathrm{bp}$. (b) Exact barcode matching, 2 nucleotide mismatch in primer matching, reads containing ambiguous characters 
were removed. (c) Only sequences that overlap longer than $10 \mathrm{bp}$ were assembled according to their overlap sequence. Reads which could not be assembled were discarded. Operational units (OTUs) were clustered with $97 \%$ similarity cutoff using UPARSE (version $7.1 \mathrm{http} / / /$ drive5.com/uparse/) and chimeric sequences were identified and removed using UCHIME. The taxonomy of each $16 \mathrm{~S}$ rDNA sequence was analyzed by RDP Classifier (http://rdp.cme.msu.edu/) against the silva (SSU123)16S rRNA database using confidence threshold of $70 \%$.

\section{Statistical analysis}

Data were analyzed using the unpaired t-test and shown as the mean \pm SD. Statistical significance was accepted at $P<0.05$. Graphic design with statistics was drawn by Graph Pad Prism 5. Pearson correlation was used to evaluate the relationship between gut microbial genera abundance and physiological parameters. Statistical significance was set at $P<0.05$.

\section{Results}

\section{Effects of WMP on body weight gain}

After 12 weeks of administration, the weight of HFD group increased by $51.53 \%$, while that of WMPtreated HFD-fed rats increased by $10.91 \%$, which was lower than that of group HFD significantly $(P<$ 0.01). WMP could significantly inhibit the increase of body weight induced by high-fat diet (Fig. 1), which was no difference of the amount of diet. Compared with group NCD, the Lee's index, epididymal fat index, perirenal fat of HFD group increased significantly $(P<0.01)$, and WMP could significantly reverse the increases (Tab.1).

\section{Effect of WMP on serum lipid profile}

In 12 weeks after administration, the HFD rats appeared hyperlipidemia as evidenced by increases of serum TC, TG and LDL-C $(P<0.01)$. The WMP rats decreased serum TC by $51.54 \%$, TG by $24.97 \%$, LDL-C by33.82\% compared with the HFD rats. The serum ASL and AST levels in group HFD were significantly increased, which was related to liver injury. The WMP group reduced AST by $26.66 \%$, ALT by $59.47 \%$.The HFD rat's MDA and NO content increased compared with NCD group $(P<0.001)$, WMP reduced MDA and NO content compared with HFD group significantly $(P<0.01)$. Furthermore, the HFD decreased expression of several genes involved with regulating lipid metabolism, including adiponectin, PPAR-y, visfatin, CEBPa and increased expression of Leptin. WMP suppressed the increase of leptin expression and increased in PPAR-y $(P<0.05)$, visfatin $(P<0.05)$, and CEBPa $(P<0.001)$ expression with the tendency of WMP to return adiponectin to normal (Tab. 2).

\section{Effect of WMP on hepatic steatosis, oxidative stress and lipid metabolism}

After 12 weeks of WMP administration, liver weight was decreased (Tab. 1) while concomitantly lowering hepatic TC and TG deposition and ameliorating HFD-induced hypercholesterolaemia and hypertriglyceridaemia in these rats. WMP treatment enhanced hepatic lipase LPL, HSL activities in rats $(P$ 
$<0.01)$. Furthermore, the leptin expression was elevated, and adiponectin, PPAR- $y$, visfatin expression reduced in HFD-fed rats compared with NCD rats. Whereas, WMP supplementation increased adiponectin, PPAR-y, visfatin expression compared with HFD rats, but no effect on leptin (Tab. 3).

The livers of NCD and WMP groups presented reddish brown and normal in shape, while the HFD group appeared yellow brown color and larger liver (Fig. 2a, upper). The HE staining results showed that the liver cells in NCD rats were arranged orderly and complete with no obvious vacuolated lipid droplets. In HFD rats, the liver tissue was irregular in the cells, the edges were not clear, and there were a large number of vacuolated lipid droplets and severe fat degeneration. Compared with the HFD group, WMP mitigated the fatty degeneration of the liver and improved the cell boundaries. It is obvious that the arrangement of hepatic cell cord tends to be normal (Fig. 2a, below). The amount of epididymal adipocytes in group HFD was significantly larger than that in group NCD and group WMP (Fig. 2b).

\section{Effect of WMP on high fat diet induced insulin resistance}

All experimental rats appeared a significant increase of blood glucose values at 30 and 60 min after oral glucose $(P<0.01)$ (Fig. 3). After $120 \mathrm{~min}$, the glycemic profile of HFD group was not reestablished to basal levels $(0 \mathrm{~min})$ and was higher than other groups significantly $(P<0.01)$. WMP administration in HFD-induced rats significantly decreased postprandial glucose levels compared with HFD group. The area under the curve for OGTTs was the largest in the HFD group, and the WMP group significantly reduced $(P<0.01)$. WMP-treated HFD-fed rats displayed improved fasting glycaemia. At the same time, WMP-treatment reduced level of insulin $(P<0.05)$ and improved insulin sensitivity. This conclusion was also supported by the lower HOMA-IR indexes of the WMP-treated HFD-fed rats when compared with their vehicle-treated HFD controls $(P<0.01)$.

\section{WMP regulated the gut microbiota}

To evaluate changes in the gut bacterial community induced by WMP, 16S rDNA from variable regions V3-V4 of the fecal samples from NCD, HFD and WMP groups were sequenced by Illumina HiSeq/MiSeq platforms; richness and evenness were calculated. At the OTU level, the HFD rats had reduced number of species when compared with the NCD rats. Diversity measured by Shannon's richness index reduced significantly in the HFD group when compared with the NCD group. The WMP rats had increased Shannon's indexes when compared with the HFD group, but had not reached the same level as the NCD group. Diversity measured by Simpson's evenness index also appeared increased values in the HFD group when compared with the NCD group, while that in the WMP group was lower than in the HFD group, but did not reach the levels as in the NCD group (Fig. 4a). The similarity of intestinal microbiota in each fecal sample was evaluated by principal component analysis (PCA). From the PC1 direction, the NCD group is closer to the WMP group than the HFD group (Fig. 4b). Heatmap expressed the first 50 species of the OUT level, and we observed that some species were restored to the NCD group by WMP (Fig. 4c).

At the phylum level, the major bacterial communities were Bacteroidetes, Firmicutes and Proteobacteria (Fig. 5a, left), and the HFD increased the relative abundance of Fusobacteria and Proteobacteria 
significantly $(P<0.05)$ against the NCD group, which could be significantly reversed by WMP (Fig. 5a, right). The WMP treatment for 12 weeks decreased in the relative abundance of Fusobacteria and Proteobacteria significantly (Fig. 5a, right), while there was a significant increase in the relative abundance of Bacteroidetes, Cyanobacteria and Actinobacteria (Fig. 5b).

At the family level, the main bacterial communities were Lachnospuraceae, Bacteroidaceae, Prevotellaceae, BacteroidalesS24-7 group, Ruminococcaceae, Lactobacillaceae (Fig. 5b, left), and the HFD diet leaded to a sharp increase in Bacteroidaceae, Peptostreptococcaceae, Fusobacteriaceae and decrease in Lactobacillaceae, while the WMP-treated HFD-fed rats reversed these change (Fig. 5b). Increased Enterobacteriaceae, Bacteroidales S24-7 group, Ruminococcaceae and Prevotellaceae were also observed in the WMP group when compared with the HFD group (Fig. 5b, right).

At the genus level, 7 top relative abundance bacteria were as follows: Bacteroides, norank Bacteroidales S24-7 group, Lactobacillus, Romboutsia, unclassified Lachnospiraceae, Prevotella-9, and 5 bacteria were with significant differences (Fig.5c). The HFD diet leaded to a sharp increase in Bacteroides, Romboutsia, Fusobacterium and decrease in norank Bacteroidales S24-7 group and Lactobaciius, while the WMPtreated HFD-fed rats reversed these changes, decreased Fusobacterium by $98.68 \%$, Bacteroides by $69.91 \%$ when compared with the HFD group (Fig. 5c, right).

At the species level, that WMP significantly reversed the increase of Fusobacterium varium, Bacteroides vulgates significantly $(P<0.01)$ and the decrease of Lachnospiraceae, Lactobacillus animalis and Akkermansia sp. (Fig. 5d).

\section{Discussion}

Although previous studies showed that WMP reduced lipid accumulation in mice and inhibited adipogenesis in 3T3-L1 cells in vitro [20], the mechanism underlying this effect and whether the WMP may modulate the composition of the gut microbiota or intestinal integrity remained unclear. This study showed that WMP not only decreased body weight gain, mitigated liver steatosis and insulin resistance but also reversed of changes in intestinal flora in HFD induced obese rats.

Our study showed that WMP treatment effectively controlled HFD-induced weight gain, reduced Lee's and visceral adiposity, moreover, the food intake of rats in each group was observed without difference, was mostly linked to reduced energy efficiency. WMP gavage fully reversed lipid accumulation in serum and liver, accompanied by the decrease of the content of MDA and the improvement of the activity of CAT and $\mathrm{GSH}$, which is in accordance with antioxidant defense mechanisms by polyphenols [12,22]. Lipotoxicity in HFD can result in increased activity levels of alanine transaminase (ALT) and aspartate transaminase (AST), which are usually detected clinical biomarkers of liver health [23], while HFD-fed rats supplemented with WMP have appeared a lower activity level of these two enzymes, and reversed hepatic steatosis derived from dyslipidemia and hepatic triglyceride accumulation. 
To explore which adipose related factors could be modulated by WMP, we detected the expression levels of leptin (LEP), adiponectin, PPAR-y, visfatin, CEBPa. The main physiological function of LEP is to regulate appetite and pathway disorders [24], and adiponectin is an adipocyte-derived hormone that acts as an antiatherogenic, antidiabetic, and anti-inflammatory[25], and accordingly, WMP supplementation reduced leptin expression and increased adiponectin expression. In the WMP-treated group, the content of visfatin in the serum and liver increased significantly, which it was largely synthesized and secreted by granulocytes and found to be an insulin sensitizer [26,27]. PPAR-y is a ligand-inducible transcription factor, it belongs to the nuclear hormone receptor superfamily that is well-known to control expression of genes involved in lipid metabolism, adipocyte differentiation and insulin sensitivity. Activation of PPAR-y can effectively improve insulin resistance, selectively promote FFA uptake by fat, inhibit liver synthesis of fatty acids, and improve lipid metabolism disorders [25,28]. PPAR- $\gamma$ is also proved to have strong antioxidant and anti-inflammatory effects. In our study, the expression of PPAR- $\gamma$ was downregulated in HFD rats, which was consistent with the reports of Natalia [29] and the WMP reversed this effect, upregulated PPAR-y to levels of the NCD rats. FAS is a key enzyme of lipid biosynthesis, which inhibiting FAS causes a decrease of fat accumulation [30]. We observed that WMP supplementation enhance the expression of enzymes involved in lipolysis and reduction in fat storage (LPL, HSL) in liver of HFD-fed rats. LPL is an enzyme that helps adipose tissue to obtain free fatty acids from triglycerides circulating in lipoproteins [31], and HSL induces lipolysis by hydrolyzing intracellular tri- and diacylglycerol. The alleviation of insulin resistance in HFD rats by WMP was evidently related to the regulation of these adipocytokines.

Intestinal flora plays a key role in the development of obesity, mainly due to the metabolic endotoxemia produced by LPS. Some studies showed that [32], on the one hand, the increase of Gram-negative strains led to a large amount of LPS formation in the intestinal tract, on the other hand, the imbalance in the proportion of bacteria inhibited the expression of intestinal tight junctions, thus significantly increased the permeability of the intestinal mucosa in mice, made a most of LPS from the intestines into the blood [33]. Short-chain fatty acids (SCFAs) are microbial fermentation products with a high concentration in the intestinal tract, which is related to glucose and lipid metabolism, secretion of gut hormones, immune homeostasis, and the physiological concentration of SCFA immediately improves the function of the colon epithelial barrier [34]. Our results showed that HFD rats increased F/B compared with NCD group, F/B in WMP-treated rats was higher than that in HFD rats. At the phylum level, WMP significantly decrease in the relative abundance of Proteobacteria and Fusobacteria. At the family level, WMP increased the SCFAs-producing bacteria, including Ruminococcaceae, Bacteroidales S24-7group, Lachnospiraceae and reduced relative abundance of Gram-negative opportunistic, such as Fusobacteriaceae, Enterobacteriaceae, Bacteroidaceae. WMP decreased the abundance of Fusobacterium, Escherichia-shigella, Bacteroides, which it belongs to Gram-negative bacteria, and increased the abundance of norank Bacteroidales S24-7 group, Lactobacilus at the genus level. Fusobacterium spp. has also been considered a pro-inflammatory passenger bacterium in the origin and proliferation of human colorectal cancer [35]. Some studies have also shown that the abundance of fecal Enterobacteriaceae induced by high fat diet increased [36]. Zhao successfully isolated Enterobacter 
cloacae B29 from the feces of a severely obese patient, it was found that the bacterial strain increased the LPS level of blood in aseptic mice, increased the level of inflammation in mice, and then presented obesity and other symptom [37]. The Lachnospiraceae family is formed by dozens of named genera, including Butyrivibrio, Ruminococcus, Anaerostipes, and Cellulosilyticum as well as a number of incertaesedis strains sharing a higher degree similarity of their $16 \mathrm{~S}$ ribosomal RNA gene sequences. All of them are strictly anaerobes. In human adults, members of this family have been associated with protection against obesity and Clostridium difficile infections. They are also known as potent SCFAs producers [38]. WMP reversed and significantly increased the abundance of Lachnospiraceae UCG005; and it also reversed and sharply raised the abundance of Akkermansia sp. in accord with that cranberry extract treatment markedly increased the proportion of the mucin-degrading bacterium Akkermansia [39].

In summary, we found that WMP treatment protected from HFD-induced obesity, liver steatosis and insulin resistance in HFD-fed rats, this effect was associated with the increase of gram-negative bacteria in the intestinal tract. At the same time, our study provided evidence for the function of walnut polyphenols in the anti-obesity, antioxidant and regulating intestinal flora.

\section{Declarations}

\section{Acknowlegements}

This study was supported by the National Natural Science Foundation of China (Grant No. 81760735; 21466037), Yunnan Biomed Major Project (2018ZF013), Yunnan Sci\& Tech Dept-YUTCM Joint Major Project (2019FF002-006) and Yunnan Province Biomedicine Major Project (202002AA100005).

\section{Ethics approval and consent to participate}

The experiment scheme was approved by the Experimental Animal Ethics Committee of Yunnan University of Traditional Chinese Medicine, Yunnan, China (IACUC permit number: R-0620170043). All experiments were performed in accordance with Regulations on the Administration of Experimental Animals (3rd, 2017), Guidelines on the Ethical Treatment of Laboratory Aanimals (2006) issued by the State Science and Technology Commission of China, and Animal Research Reporting of In Vivo Experiments (ARRIVE) guidelines. Experimental programs strive to reduce animal suffering by using only the necessary number of animals to produce reliable scientific data.

\section{Consent for publication}

Not applicable.

\section{Competing interests}

The authors declare that they have no competing interests.

\section{Author contributions}


SZ designed the overall research study and managed it. XH generated the animal experimental results; $\mathrm{XH}$ and DC were involved in analysis and interpretation of gut microbiota; SZ and DC managed this project and implemented data integration and statistical analysis; HX and CD wrote the manuscript. All authors provided input on the analysis and interpretation of the results, and preparation of the manuscript.

\section{ORCID}

Shenglan Zhao id https://orcid.org/0000-0001-8817-5996

Chaoyin Chen id https://orcid.org/0000-0003-1622-1118

\section{References}

1. Yoon KH, Lee JH, Kim JW, Cho JH, Choi YH, Ko SH, Zimmet P, Son HY. Epidemic obesity and type 2 diabetes in Asia. Lancet 2006;368(9548):1681-8.

2. Friedman JM. Modern science versus the stigma of obesity. Nat Med 2004;10(6):563-9.

3. Jang MK, Yun YR, Kim JH, Park MH, Jung MH. Gomisin N inhibits adipogenesis and prevents highfat diet-induced obesity. Scientific Reports 2017;7(40345):1-11.

4. Fan W, Xu Y, Liu Y, Zhang Z, Lu L, Ding Z. Obesity or overweight, a chronic inflammatory status in male reproductive system, leads to mice and human subfertility. Front Physiol 2017;8:1117.

5. Biddinger SB, Kahn CR. From mice to men: insights into the insulin resistance syndromes. Annu Rev Physiol 2006;68(68):123-58.

6. Utzschneider KM, Kahn SE. Review: The role of insulin resistance in nonalcoholic fatty liver disease. The Journal of Clinical Endocrinology \& Metabolism 2006;91(12):4753-61.

7. Sonnenburg ED, Smits SA, Tikhonov M, Higginbottom SK, Wingreen NS, Sonnenburg JL. Diet-induced extinctions in the gut microbiota compound over generations. Nature 2016;529(7585):212-5.

8. Parseus A, Sommer N, Sommer F, Caesar R, Molinaro A, Stahlman M, Greiner TU, Perkins R, Backhed F. Microbiota-induced obesity requires farnesoid X receptor. Gut 2017;66(3):429-437.

9. Turnbaugh PJ, Ley RE, Mahowald MA, Magrini V, Mardis ER, Gordon JI. An obesity-associated gut microbiome with increased capacity for energy harvest. Nature 2006;444(7122):1027-31.

10. Backhed F, Manchester JK, Semenkovich CF, Gordon JI. Mechanisms underlying the resistance to diet-induced obesity in germ-free mice. Proc Natl Acad Sci U S A 2007;104(3):979-84.

11. Liu Z, Brooks RS, Ciappio ED, Kim SJ, Crott JW, Bennett G, Greenberg AS, Mason JB. Diet-induced obesity elevates colonic TNF-alpha in mice and is accompanied by an activation of Wnt signaling: a mechanism for obesity-associated colorectal cancer. J Nutr Biochem 2012;23(10):1207-13.

12. Graziani G, D'Argenio G, Tuccillo C, Loguercio C, Ritieni A, Morisco F, Del VBC, Fogliano V, Romano M. Apple polyphenol extracts prevent damage to human gastric epithelial cells in vitro and to rat gastric mucosa in vivo. Gut 2005;54(2):193-200. 
13. Isokpehi RD, Simmons SS, Johnson MO, Payton M. Genomic evidence for bacterial determinants influencing obesity development. Int J Environ Res Public Health 2017;14(4):345.

14. Farr OM, Tuccinardi D, Upadhyay J, Oussaada SM, Mantzoros CS. Walnut consumption increases activation of the insula to highly desirable food cues: A randomized, double-blind, placebo-controlled, cross-over fMRI study. Diabetes Obes Metab 2018;20(1):173-177.

15. Communittee-Phannacopoeia-China. Phannacopoeia of China Vol. 1. Beijing: Sci\&Tech Press of China Medicine; 2015. 279 p.

16. Tuccinardi D, Farr OM, Upadhyay J, Oussaada SM, Klapa MI, Candela M, Rampelli S, Lehoux S, Lazaro I, Sala-Vila A and others. Mechanisms underlying the cardiometabolic protective effect of walnut consumption in obese people: A cross-over, randomized, double-blind, controlled inpatient physiology study. Diabetes Obes Metab 2019;21(9):2086-2095.

17. Luo Y, Wu W, Chen D, Lin Y, Ma Y, Chen C, Zhao S. Optimization of simultaneous microwave/ultrasonic-assisted extraction of phenolic compounds from walnut flour using response surface methodology. Pharm Biol 2017;55(1):1999-2004.

18. Martinez ML, Labuckas DO, Lamarque AL, Maestri DM. Walnut (Juglans regia L.): genetic resources, chemistry, by-products. J Sci Food Agri 2010;90(12):1959-67.

19. Blomhoff R, Carlsen MH, Andersen LF, Jacobs DJ. Health benefits of nuts: potential role of antioxidants. Br J Nutr 2006;96(Supp):52-60.

20. Liang $X$, Chen D, Cao L, Zhao S. Effects of pressed degreased walnut meal extracts on lipid metabolism in postnatally monosodium glutamate-induced mice and 3T3-L1 preadipocytes. J Funct Foods 2017;31(1):89-96.

21. Cai D, Yuan M, Frantz DF, Melendez PA, Hansen L, Lee J, Shoelson SE. Local and systemic insulin resistance resulting from hepatic activation of IKK-beta and NF-kappaB. Nat Med 2005;11(2):183-90.

22. Stagos D, Amoutzias GD, Matakos A, Spyrou A, Tsatsakis AM, Kouretas D. Chemoprevention of liver cancer by plant polyphenols. Food Chem Toxicol 2012;50(6):2155-70.

23. Kim CM, Yi SJ, Cho IJ, Ku SK. Red-koji fermented red ginseng ameliorates high fat diet-induced metabolic disorders in mice. Nutrients 2013;5(11):4316-32.

24. Sloan C, Tuinei J, Nemetz K, Frandsen J, Soto J, Wride N, Sempokuya T, Alegria L, Bugger H, Abel ED. Central leptin signaling is required to normalize myocardial fatty acid oxidation rates in caloricrestricted ob/ob mice. Diabetes 2011;60(5):1424-34.

25. Gastaldelli A, Miyazaki Y, Mahankali A, Berria R, Pettiti M, Buzzigoli E, Ferrannini E, DeFronzo RA. The effect of pioglitazone on the liver: role of adiponectin. Diabetes Care 2006;29(10):2275-81.

26. Mu J, Feng B, Ye Z, Yuan F, Zeng W, Luo Z, Qi W. Visfatin is related to lipid dysregulation, endothelial dysfunction and atherosclerosis in patients with chronic kidney disease. J Nephrol 2011;24(2):17784.

27. Stastny J, Bienertova-Vasku J, Vasku A. Visfatin and its role in obesity development. Diabetes Metab Syndr 2012;6(2):120-4. 
28. Ahmadian M, Suh JM, Hah N, Liddle C, Atkins AR, Downes M, Evans RM. PPARgamma signaling and metabolism: the good, the bad and the future. Nat Med 2013;19(5):557-66.

29. Ramirez NM, Toledo R, Moreira M, Martino H, Benjamin L, de Queiroz JH, Ribeiro AQ, Ribeiro S. Antiobesity effects of tea from Mangifera indica L. leaves of the Uba variety in high-fat diet-induced obese rats. Biomed Pharmacother 2017;91:938-945.

30. Liang Y, Tian W, Ma X. Inhibitory effects of grape skin extract and resveratrol on fatty acid synthase. BMC Complement Altern Med 2013;13:361.

31. Wang H, Eckel RH. Lipoprotein lipase: from gene to obesity. Am J Physiol Endocrinol Metab 2009;297(2):E271-88.

32. Cani PD, Amar J, Iglesias MA, Poggi M, Knauf C, Bastelica D, Neyrinck AM, Fava F, Tuohy KM, Chabo $\mathrm{C}$ and others. Metabolic endotoxemia initiates obesity and insulin resistance. Diabetes 2007;56(7):1761-72.

33. Chang CJ, Lu CC, Lin CS, Martel J, Ko YF, Ojcius DM, Wu TR, Tsai YH, Yeh TS, Lu JJ and others. Antrodia cinnamomea reduces obesity and modulates the gut microbiota in high-fat diet-fed mice. Int J Obes (Lond) 2018;42(2):231-243.

34. Roager HM, Vogt JK, Kristensen M, Hansen L, lbrugger S, Maerkedahl RB, Bahl MI, Lind MV, Nielsen $\mathrm{RL}$, Frokiaer $\mathrm{H}$ and others. Whole grain-rich diet reduces body weight and systemic low-grade inflammation without inducing major changes of the gut microbiome: a randomised cross-over trial. Gut 2019;68(1):83-93.

35. Tjalsma H, Boleij A, Marchesi JR, Dutilh BE. A bacterial driver-passenger model for colorectal cancer: beyond the usual suspects. Nat Rev Microbiol 2012;10(8):575-82.

36. Martinez-Medina M, Denizot J, Dreux N, Robin F, Billard E, Bonnet R, Darfeuille-Michaud A, Barnich N. Western diet induces dysbiosis with increased E coli in CEABAC10 mice, alters host barrier function favouring AIEC colonisation. Gut 2014;63(1):116-24.

37. Fei N, Zhao L. An opportunistic pathogen isolated from the gut of an obese human causes obesity in germfree mice. ISME J 2013;7(4):880-4.

38. Sagheddu V, Patrone V, Miragoli F, Puglisi E, Morelli L. Infant early gut colonization by Lachnospiraceae: high frequency of Ruminococcus gnavus. Front Pediatr 2016;4:57.

39. Anhe FF, Roy D, Pilon G, Dudonne S, Matamoros S, Varin TV, Garofalo C, Moine Q, Desjardins Y, Levy $E$ and others. A polyphenol-rich cranberry extract protects from diet-induced obesity, insulin resistance and intestinal inflammation in association with increased Akkermansia spp. population in the gut microbiota of mice. Gut 2015;64(6):872-83.

\section{Tables}

Table 1 Effect of WMP on visceral index of rats 


\begin{tabular}{|llll|}
\hline & NCD & HFD & WMP \\
\hline Weight(g) & $439.91 \pm 36.95$ & $631.11 \pm 59.06^{\# \# \#}$ & $466.82 \pm 42.71^{\star \star \star}$ \\
\hline Lee's index(\%) & $25.26 \pm 0.55$ & $28.37 \pm 0.74^{\# \# \#}$ & $26.14 \pm 0.62^{\star \star \star}$ \\
\hline Liver index(\%) & $2.24 \pm 0.18$ & $3.57 \pm 0.56^{\# \# \#}$ & $2.73 \pm 0.28^{\star \star \star}$ \\
\hline Epididymal adipose index (\%) & $1.36 \pm 0.25$ & $2.08 \pm 0.44^{\# \#}$ & $1.39 \pm 0.42^{\star \star}$ \\
\hline Perirenal adipose index (\%) & $1.57 \pm 0.45$ & $3.07 \pm 0.81^{\# \# \#}$ & $1.78 \pm 0.76^{\star \star \star}$ \\
\hline
\end{tabular}

Rats were fed either a normal or a high-fat diet (HFD) for 12 weeks HFD-fed animals were treated with daily oral doses of WMP $(750 \mathrm{mg} / \mathrm{kg})$ normal and HFD fed control rats were gavaged with vehicle (normal saline). Data are expressed as the mean \pm SD $(n=10)$. ${ }^{\#} P<0.01$ and ${ }^{\# \#} P<0.001$ compared with NCD group; $* * ~ P<0.01$ and ${ }^{* \star} P<0.001$ compared with HFD group.

Table 2 Effect of WMP on the serum parameters.

\begin{tabular}{|c|c|c|c|}
\hline & NCD & HFD & WMP \\
\hline $\mathrm{TC}(\mathrm{mmol} / \mathrm{l})$ & $0.59 \pm 0.14$ & $1.38 \pm 0.27^{\# \# \#}$ & $0.67 \pm 0.14^{\star \star \star}$ \\
\hline $\mathrm{TG}(\mathrm{mmol} / \mathrm{l})$ & $0.56 \pm 0.21$ & $1.18 \pm 0.23^{\# \# \#}$ & $0.89 \pm 0.21^{\star \star \star}$ \\
\hline LDL-C(mmol/l) & $0.45 \pm 0.12$ & $1.21 \pm 0.23^{\# \# \#}$ & $0.80 \pm 0.23^{\star \star \star}$ \\
\hline $\mathrm{HDL}-\mathrm{C}(\mathrm{mmol} / \mathrm{l})$ & $0.73 \pm 0.38$ & $0.65 \pm 0.11$ & $0.78 \pm 0.24$ \\
\hline $\mathrm{MDA}(\mathrm{nmol} / \mathrm{ml})$ & $4.23 \pm 0.48$ & $7.04 \pm 0.94^{\# \# \#}$ & $3.82 \pm 0.33^{\star \star \star}$ \\
\hline Leptin(ng/ml) & $14.43 \pm 1.94$ & $17.80 \pm 1.33^{\# \#}$ & $15.04 \pm 2.11^{*}$ \\
\hline Visfatin(ng/ml) & $49.58 \pm 13.93$ & $37.63 \pm 4.06$ & $53.94 \pm 12.26^{*}$ \\
\hline PPAR-y(ng/ml) & $5.93 \pm 2.18$ & $2.67 \pm 1.06^{\# \# \#}$ & $5.21 \pm 1.41^{*}$ \\
\hline AST(IU/L) & $12.10 \pm 2.45$ & $20.14 \pm 3.97^{\# \# \#}$ & $14.77 \pm 4.48^{\star \star \star}$ \\
\hline $\mathrm{ALT}(\mathrm{IU} / \mathrm{L})$ & $7.67 \pm 5.24$ & $29.12 \pm 3.60^{\# \# \#}$ & $11.80 \pm 5.02^{\star \star \star}$ \\
\hline $\mathrm{NO}(\mu \mathrm{mol} / \mathrm{l})$ & $6.45 \pm 1.49$ & $10.18 \pm 2.79 \# \#$ & $6.74 \pm 2.37^{\star \star}$ \\
\hline Adiponectin( $\mu \mathrm{g} / \mathrm{ml})$ & $1.17 \pm 0.32$ & $0.57 \pm 0.12^{\# \#}$ & $0.83 \pm 0.26$ \\
\hline CEBPa(ng/ml) & $3.59 \pm 0.35$ & $2.56 \pm 0.26^{\# \# \#}$ & $3.26 \pm 0.37^{\star \star \star}$ \\
\hline
\end{tabular}


Note: Rats were fed either a normal or a high-fat diet (HFD) for 12 weeks HFD-fed animals were treated with daily oral doses of WMP $(750 \mathrm{mg} / \mathrm{kg})$ normal and HFD fed control rats were gavaged with vehicle (normal saline). Data are expressed as the mean $\pm S D(n=10) .{ }^{\#} P<0.01$ and ${ }^{\# \# \#} P<0.001$ compared with NCD group; ${ }^{*} P<0.05,{ }^{* \star} \mathrm{P}<0.01$ and ${ }^{\star \star *} P<0.001$ compared with HFD group.

Table 3 Effect of WMP on hepatic steatosis, oxidative stress and lipid metabolism.

\begin{tabular}{|c|c|c|c|}
\hline & NCD & HFD & WMP \\
\hline $\mathrm{TC}(\mu \mathrm{mol} / \mathrm{g})$ & $1.01 \pm 0.18$ & $4.78 \pm 1.25^{\# \# \#}$ & $1.65 \pm 0.43^{\star \star \star}$ \\
\hline $\mathrm{TG}(\mu \mathrm{mol} / \mathrm{g})$ & $3.76 \pm 0.81$ & $9.04 \pm 2.40^{\# \# \#}$ & $5.36 \pm 1.75^{\star \star \star}$ \\
\hline $\mathrm{LDL}-\mathrm{C}(\mu \mathrm{mol} / \mathrm{g})$ & $2.14 \pm 1.43$ & $4.88 \pm 2.44^{\# \# \#}$ & $1.81 \pm 0.56^{\star \star \star}$ \\
\hline $\mathrm{HDL}-\mathrm{C}(\mu \mathrm{mol} / \mathrm{g})$ & $0.38 \pm 0.05$ & $0.13 \pm 0.08^{\# \# \#}$ & $0.37 \pm 0.07^{\star \star \star}$ \\
\hline $\operatorname{MDA}(\mu \mathrm{mol} / \mathrm{g})$ & $4.66 \pm 0.74$ & $5.76 \pm 0.32$ & $4.29 \pm 0.74^{\star \star \star}$ \\
\hline Leptin( $\mu \mathrm{g} / \mathrm{g})$ & $0.19 \pm 0.06$ & $0.38 \pm 0.11^{\# \# \#}$ & $0.28 \pm 0.05^{\star}$ \\
\hline Visfatin(ng/g) & $9.15 \pm 2.14$ & $2.69 \pm 0.52^{\# \# \#}$ & $11.99 \pm 2.03^{\star \star \star}$ \\
\hline PPAR- $\gamma(\mu \mathrm{g} / \mathrm{g})$ & $7.16 \pm 1.27$ & $5.20 \pm 1.10^{\# \# \#}$ & $10.51 \pm 0.88^{\star \star \star}$ \\
\hline Prot(g/kg) & $1.85 \pm 0.23$ & $1.46 \pm 0.07^{\# \# \#}$ & $1.78 \pm 0.14^{\star \star \star}$ \\
\hline $\mathrm{GSH}(\mathrm{mg} / \mathrm{g}$ prot$)$ & $1.01 \pm 0.18$ & $0.77 \pm 0.071^{\# \# \#}$ & $1.24 \pm 0.15^{\star \star \star}$ \\
\hline CAT(U/ugprot) & $0.92 \pm 0.12$ & $0.75 \pm 0.12^{\#}$ & $0.94 \pm 0.16^{\star *}$ \\
\hline LPL(pg/g) & $52.24 \pm 13.06$ & $15.54 \pm 2.50^{\# \# \#}$ & $44.01 \pm 6.67^{\star \star \star}$ \\
\hline $\mathrm{HSL}(\mathrm{mU} / \mathrm{g})$ & $116.6 \pm 24.74$ & $43.90 \pm 8.09^{\# \# \#}$ & $140.40 \pm 7.94^{\star * *}$ \\
\hline
\end{tabular}

Note: Rats were fed either a normal or a high-fat diet (HFD) for 12 weeks. HFD-fed animals were treated with daily oral doses of WMP $(750 \mathrm{mg} / \mathrm{kg})$ while normal and HFD fed control rats were gavaged with vehicle (normal saline). Data are expressed as the mean \pm SD $(n=10) .{ }^{\#} P<0.05,{ }^{\#} P<0.01$ and ${ }^{\# \# \#} P<$ 0.001 compared with NCD group; * $P<0.05$, ** $P<0.01$ and $* \star \star ~ P<0.001$ compared with HFD group.

\section{Figures}


a

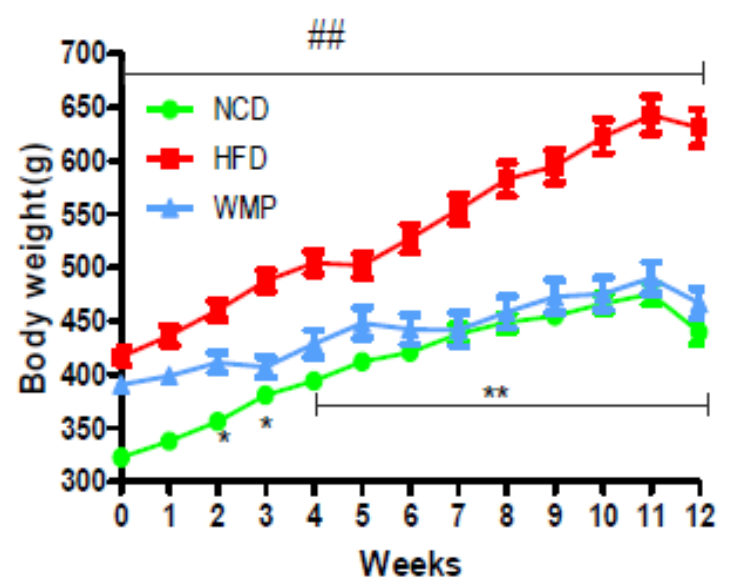

$b$

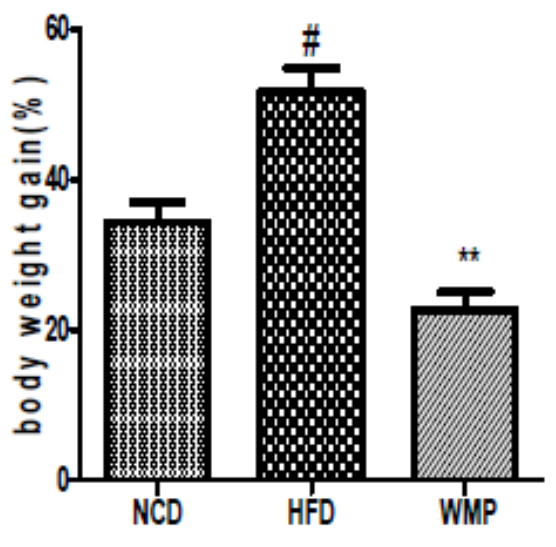

$c$

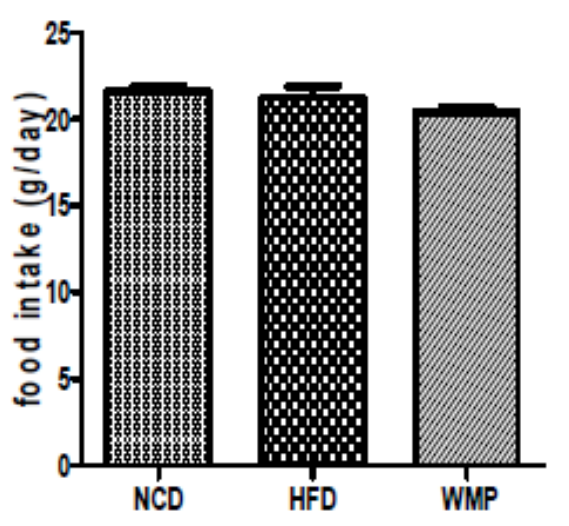

Figure 1

Fig. 1 Effect of WMP on the body weight of HFD-fed rats. Rats were fed either a normal or a high-fat diet (HFD) for 12 weeks. HFD-fed animals were treated with daily oral doses of WMP $(750 \mathrm{mg} / \mathrm{kg})$ while Chow and HFD fed control rats were gavaged with vehicle (normal saline), (a) body weight, (b) total weight gain, (c) food intake. Data are expressed as the mean $\pm S D(n=10)$. \# $P<0.05$ and \#\# $P<0.01$ HFD vs. NCD; * $P$ $<0.05$ and $* * P<0.01$ WMP vs. HFD. 
a
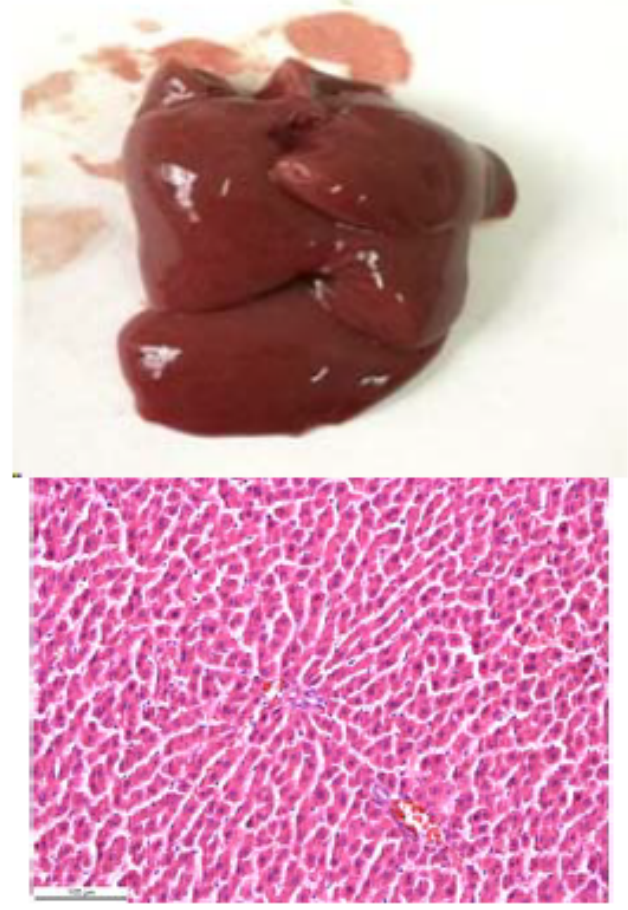

HFD
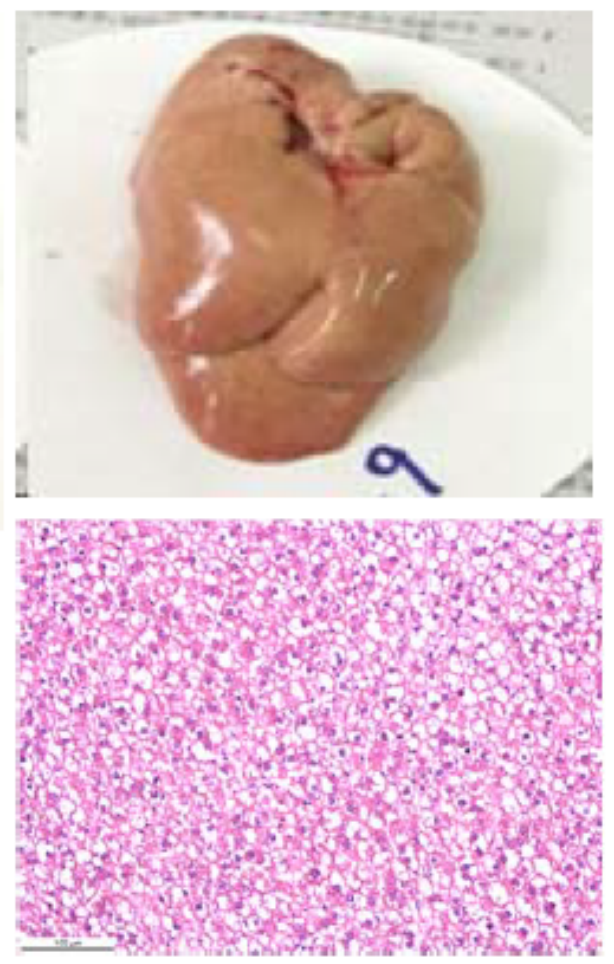

WMP
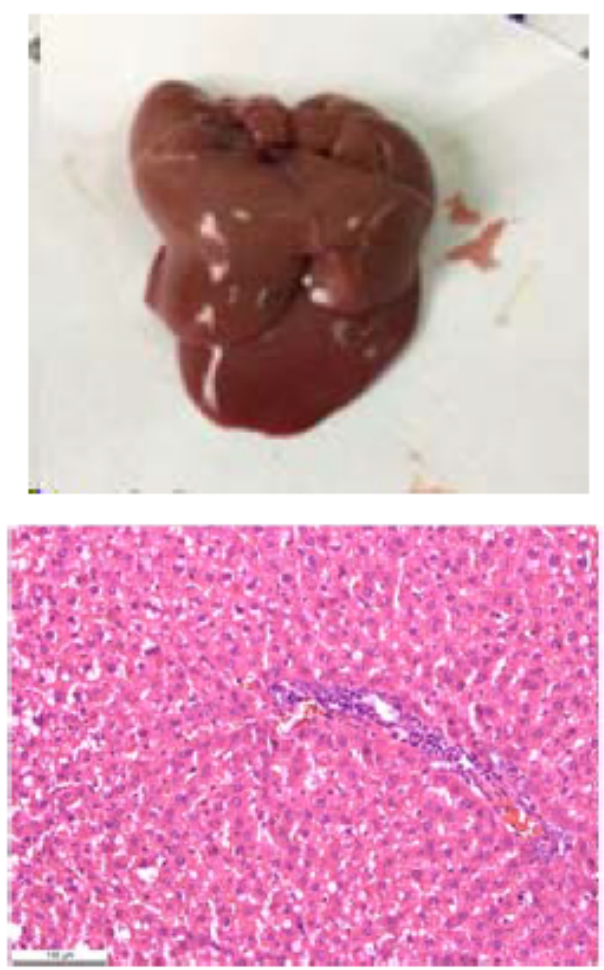

b
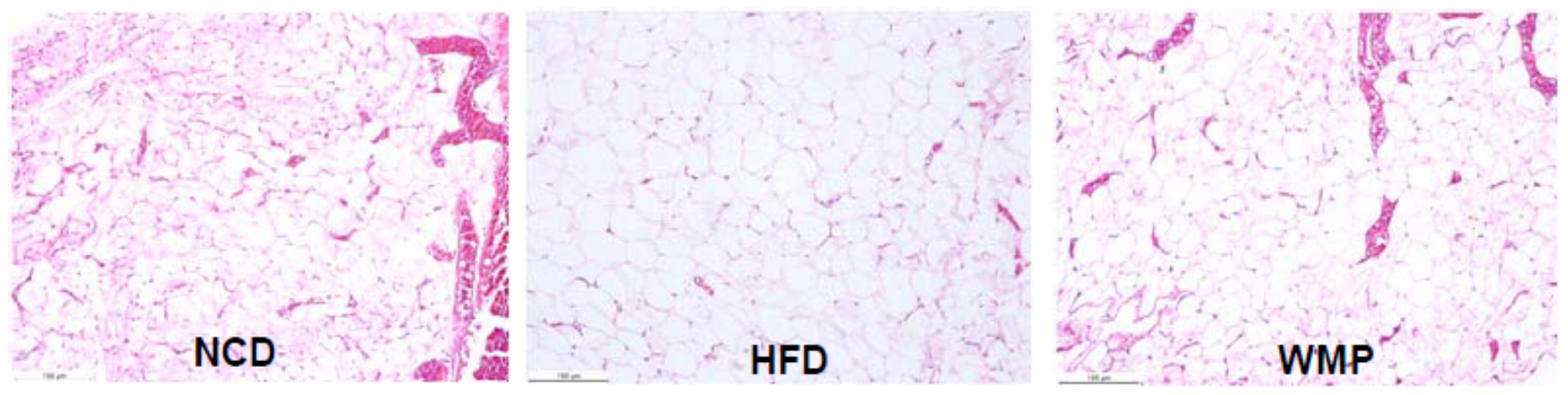

Figure 2

HE staining of liver and epididymal adipose tissue in rats. Rats 3 were fed either a normal or a high-fat diet (HFD) for 12 weeks HFD-fed animals were treated with daily oral doses of WMP $(750 \mathrm{mg} / \mathrm{kg})$ normal and HFD fed control rats were gavaged with vehicle (normal saline). (a)Liver tissue morphology (upper) and HE staining (below, 200x). (b) Adipocyte morphology was assessed by H\&E staining (400x). 

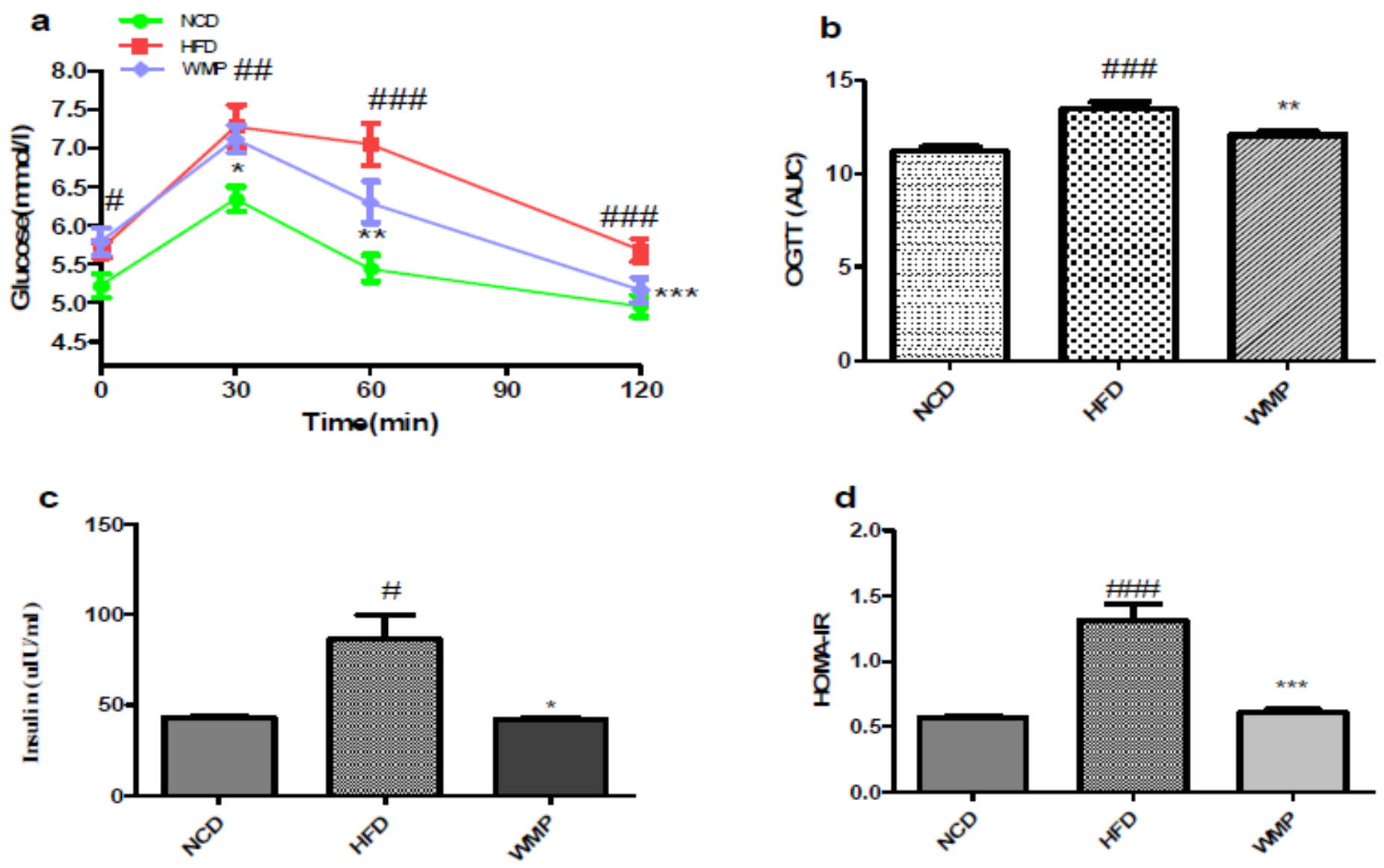

Figure 3

Effect of WMP on insulin-glucose axis function. The effect 3 of WMP on the function of the Rats were 12 $\mathrm{h}$ fasted for fasting glycaemia (a) Changes in tail blood glucose during Oral glucose tolerance test (OGTT). (b) Area under the curve for OGTTs. (c) Fasting serum insulin levels. (d) HOMA-IR, homeostasis model index for insulin resistance (IR). Data are expressed as the mean $\pm S E M(n=10)$. \# $P<0.05$, \#\# $P<$ 0.01 and \#\#\# $\mathrm{P}<0.001$ compared with NCD group; * $\mathrm{P}<0.05$, ${ }^{\star \star} \mathrm{P}<0.01$ and ${ }^{\star \star \star} \mathrm{P}<0.001$ compared with HFD group 
a
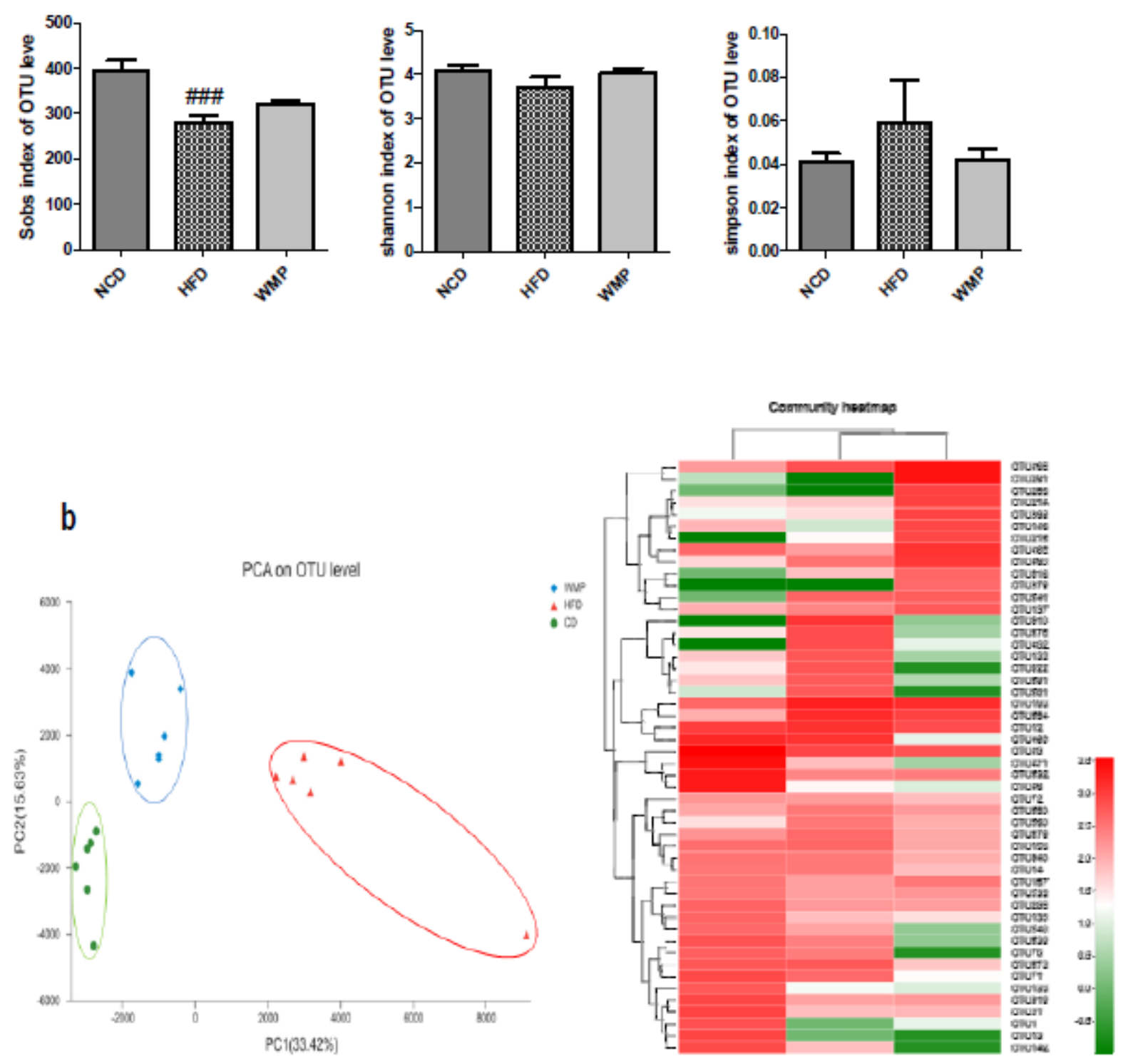

Figure 4

WMP modulated the structure and diversity of 3 the gut microbiota Rats were fed either a normal or a high-fat diet (HFD) for 12 weeks. HFD-fed animals were treated with daily oral doses of WMP (750 $\mathrm{mg} / \mathrm{kg}$ ) while normal and HFD fed control rats were gavaged with vehicle (normal saline). (a)Alpha diversity analysis at the OUT level calculated on denoised sequences of rat cecal microbiota (b) principal component analysis (c) heatmap on OUT level. Data are expressed as the mean $\pm S D(n=8)$. \#\#\# $P<0.001$ compared with NCD group. 
(a)

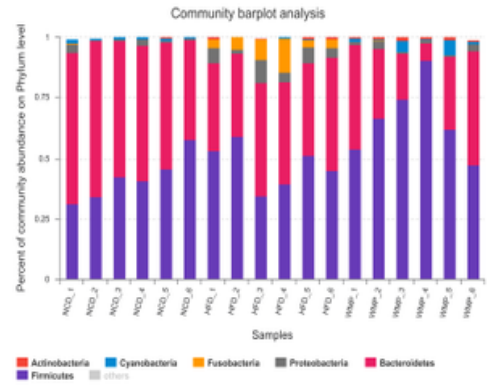

(b)

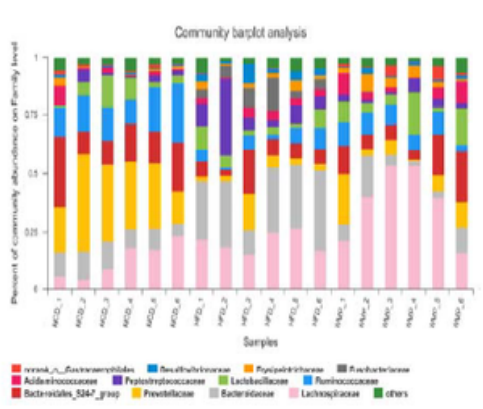

(c)
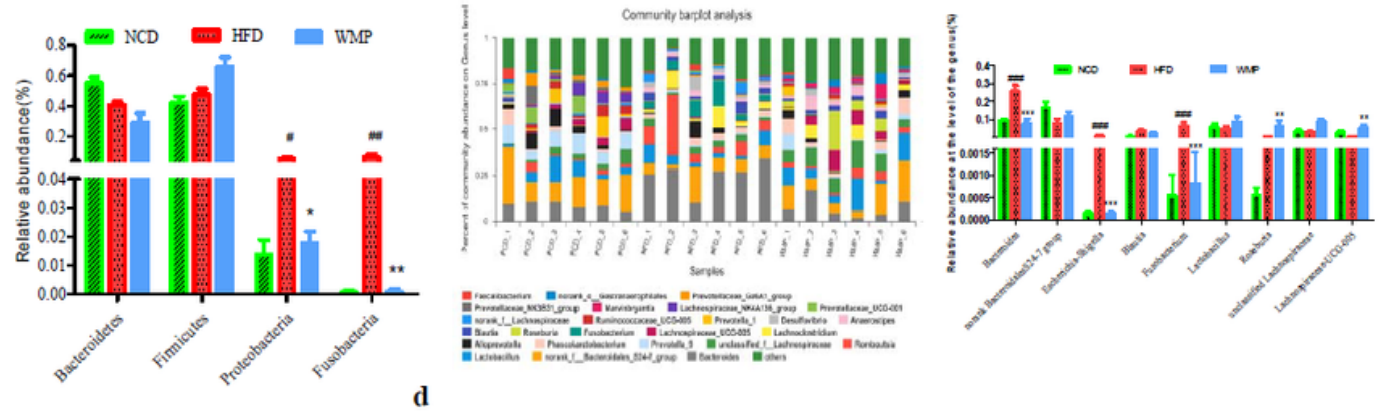

d
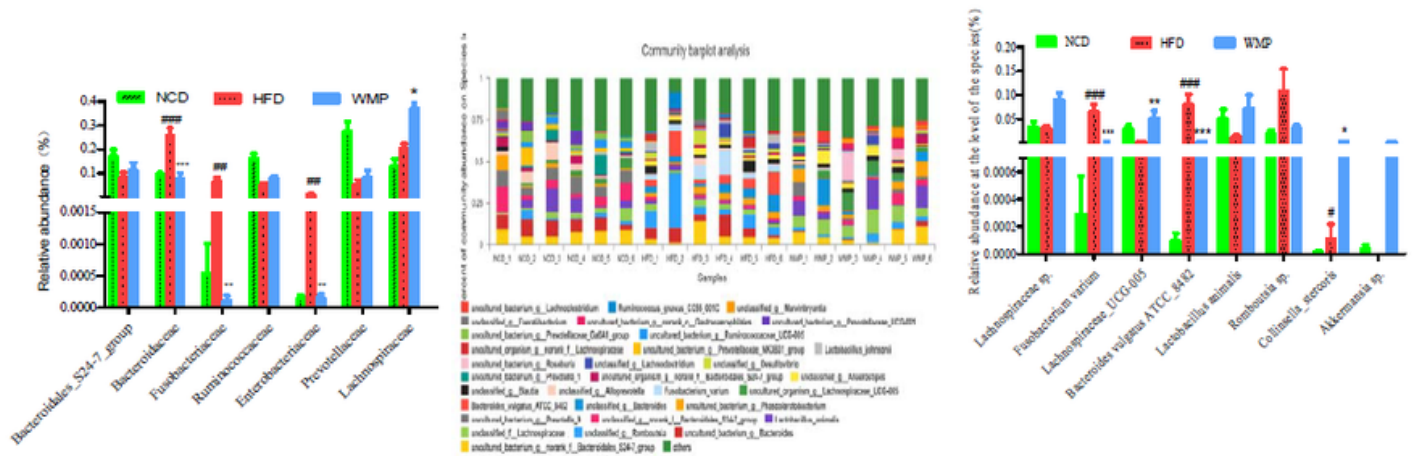

Figure 5

WMP modulated the composition of the gut microbiota. Rats 2 were fed either a normal or a high-fat diet (HFD) for 12 weeks. HFD-fed animals were treated with daily oral doses of WMP $(750 \mathrm{mg} / \mathrm{kg}$ ) while normal and HFD fed control rats were gavaged with vehicle (normal saline). (a) phylum-level, (b) familylevel, (c) genus-level, (d) species-level. The left graph is the community composition of all the samples, the right graph shows significant differences in the flora. Data are expressed as the mean $\pm S D(n=8)$. \# $P$ $<0.05$, \#\# $P<0.01$ and \#\#\# $P<0.001$ compared with NCD group; $* P<0.05$, $* \star P<0.01$ and $* \star \star ~ P<0.001$ compared with HFD group. 